\title{
Mutation-Induced Conformational Changes and Energetics for Binding of FMN Ligand in Flavin Mononucleotide Riboswitch by Molecular Dynamics Simulations
}

\author{
Padmaja D. Wakchaure, ${ }^{1,2}$ Bishwajit Ganguly ${ }^{1,2, *}$
}

\footnotetext{
${ }^{1}$ Computation and Simulation Unit (Analytical Discipline and Centralized Instrument Facility), CSIR-Central Salt and Marine Chemicals Research Institute, Bhavnagar-364002, Gujarat, India

2 Academy of Scientific and Innovative Research, CSIR-CSMCRI, Bhavnagar-364002, Gujarat, India

* Corresponding author's e-mail address: ganguly@csmcri.res.in
}

RECEIVED: July 17, 2019 * REVISED: August 17, 2019 * ACCEPTED: August 31, 2019

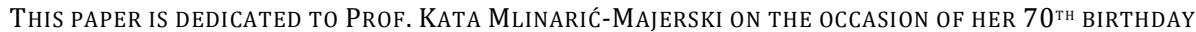

\begin{abstract}
Riboswitches are the type of regulatory elements present in the untranslated region of mRNA and specifically bind to the natural ligand to regulate gene expression. This binding specificity can be affected by even single point mutation incorporated in the core of the riboswitch. In this work, we have examined the mutations at the binding site residue in Flavin Mononucleotide (FMN) riboswitch structure with 30ns molecular dynamics simulations. The interaction of ligand (FMN) with riboswitch has been characterized using root mean square deviation, hydrogen bonding analysis, and the calculated binding affinities. Mutation at A48G and G62U show the enhanced binding energy however, the mutation at A85G, are energetically unfavorable compared to the wild type. This work gives valuable insight into the structures and energetics of the mutated FMN riboswitch to design new hits for biological applications.
\end{abstract}

Keywords: FMN riboswitch, mutation, MD simulations.

\section{INTRODUCTION}

$\mathbf{R}$ IBOSWITCHES are RNA elements present in 5' untranslated regions in mRNA that regulates the expression of downstream genes in response to specific metabolites. ${ }^{[1]}$ Riboswitches are emerging as a new target for antibiotics design. ${ }^{[2]}$ In recent literature, there are several reports on ligand design for different types of riboswitches for various applications. ${ }^{[3]}$ Among these different types of riboswitches, ligands such as roseoflavin and 5FDQD binding to Flavin mononucleotide (FMN) riboswitch found to be useful for antibacterial activity in vivo. ${ }^{[4-6]}$ Ligands like ribocil ${ }^{[7]}$ and flavin analogs ${ }^{[2]}$ were reported for targeting the FMN riboswitch. $X$-ray crystal structure study shows a butterfly fold made up of two domains P2-P6 and P3-P5 joined together by L2-L6 loops. ${ }^{[8]}$ Isoalloxazine moiety in FMN is sandwiched between Adenine (A) 48 and A85. The uracil ring in isoalloxazine shows hydrogen bonding with A99. Only one of the three hydroxyl groups in the ribityl- phosphate chain in FMN forms hydrogen bonds with Guanine (G) 11 and phosphate group forms magnesium (Mg) bridged interaction with G33. In addition to these interactions, the phosphate group in FMN forms hydrogen bonds with $\mathrm{G} 10, \mathrm{G} 11, \mathrm{G} 32, \mathrm{G} 62$, and $\mathrm{G} 84$. It is reported that the non $\mathrm{G}: \mathrm{C}$ base-pairing composition in riboswitch sequence affects the FMN dissociation rate constant. ${ }^{[9]}$ Gene expression in downstream genes can be tuned by mutating the base pairings in the binding site. In this study, non $G: C$ base pairs were mutated in P3-P6 regions. Mutation of A85:U94 base pair with $\mathrm{G}: \mathrm{C}$ base-pair have shown the highest binding affinity of riboswitch with the ligand. A few binding site residues like Cytosine(C)30, G47, and $\mathrm{G} 70$ have also been studied by mutation and $\mathrm{G} 47$ found to be critical in aligning the adjacent adenine confirmation for stacking with FMN ligand which is essential in FMN binding. ${ }^{[10]}$ All these experimental studies show that base pair composition, as well as a single base mutation in the binding site, has a direct influence on ligand binding 
parameters. Molecular dynamics (MD) simulation have been extensively used for studying the influence of mutations on binding parameters of ligands to protein and RNA structures. ${ }^{[11-15]}$ We have examined the contribution of 12 residues in the binding site for stabilizing the FMN ligand. ${ }^{[16]}$ In present work, we have examined the influence of five binding site mutations on FMN ligand binding and stability of riboswitch structure. The residues A-85 and A48 which sandwich the FMN ligand are replaced with guanine base. The replacement of A-85 with $G$ resulted in a purine-purine base pairing which is an important class of base pairing in RNA structure. The close similarity in the structural motif of $A \& G$ bases would not induce any deleterious steric effect in the riboswitch; however, the $\mathrm{G}$ base can provide additional functional groups like $-\mathrm{NH}$ and -CO group for favorable interaction with the ligand molecule. The residue G-62 has been replaced with uracil $(U)$ to examine the effect of replacing the purine with pyrimidine moiety. Further, the residues G- 84 and G- 33 are replaced with $A$, in such cases, the number of hydrogen bond donor and acceptors would be less than the $G$ and the MD study would reveal the effect of such changes in the mutated FMN riboswitch upon interaction with ligand molecules. This study gives insights on important ligand binding interactions required for ligand stabilization for the design of new ligands for better binding affinity with FMN riboswitch.

\section{COMPUTATIONAL METHODOLOGY}

\section{System Preparation}

Atomic coordinates were obtained from RCSB-Protein Data Bank (PDB) ${ }^{[17]}$ for FMN complex with riboswitch 2YIE. ${ }^{[18]}$ Initial preparation was performed in PyMo|[19] and addition of hydrogen and mutations were done in Discovery studio. ${ }^{[20]}$ The ligand topology files were prepared with the ACPYPE-Antechamber Python Parser interface and AM1BCC charges were used. ${ }^{[21,22]}$

\section{Molecular Dynamics Simulation}

All energy minimization and molecular dynamics simulations were performed in GROMACS-4.5.5 package with Amber99sb_ParmBSC0 force field..23-27] All the systems were kept in cubic periodic boundary condition box of size nearly $101.9 \AA \times 101.9 \AA \times 101.9 \AA$ extending $10 \AA$ from the complex. Each complex was solvated with explicit water with TIP3P water model. The crystal structure of FMN riboswitch contains $13 \mathrm{Mg}^{2+}$ ions and we considered the same number of $\mathrm{Mg}^{2+}$ ions for simulations. To neutralize the charges, 100 sodium and 20 chloride ions were added in the simulation box. All bond lengths were constrained with LINear Constraint Solver (LINCS) algorithm. ${ }^{[28]}$ The electrostatic interactions were evaluated with particlemesh Ewald (PME) scheme. ${ }^{[29]}$ Short-range electrostatic interaction and van der Waals interactions were considered with $10 \AA$ cutoff. The complex was minimized with the steepest descent method. The first phase of equilibration was performed with NVT ensemble for 200 ps at $300 \mathrm{~K}$ temperature conditions with the $\mathrm{v}$-rescale thermostat. Second equilibration step performed at 1atm pressure with NPT ensemble with Berendsen barostat. The last production run was performed for 30ns for each mutated riboswitch-ligand complex at NPT ensemble. The trajectories were analyzed and data were plotted using Origin software. ${ }^{[30]}$ The binding energies were calculated for the geometries obtained from the final trajectory of MD simulations. The binding energies were evaluated with universal force field ${ }^{[31]}$ in GAUSSIAN $09^{[32]}$ in the gas phase using the following equation.

$\Delta E=E($ RNA ligand complex $)-[E($ RNA $)+E($ ligand $)]$

The relative binding energies were calculated with respect to wild type to examine the effect of the mutation on the binding energy of FMN ligand with riboswitch.

\section{RESULTS AND DISCUSSION}

To study the preferential binding of FMN to wild type FMN riboswitch and mutated FMN riboswitch structure, we have performed the structural analysis with calculating root mean square deviation (RMSD), root means square fluctuations (RMSF), the radius of gyration and hydrogen bond interactions. The binding energy analysis was performed with the Universal force field which has also been employed for studying similar biomolecular systems. ${ }^{[33-35]}$

\section{Structural Stability of Riboswitch}

The root mean square deviation (RMSD) was calculated relative to the starting structure during the production phase to understand the effect of mutations on conformational stability. It is observed that RMSD profiles are quite similar to its starting structures and ensures that the trajectories are stable during the simulation for all complexes. The average RMSD observed for wild type $3.1 \AA$, for $A-85$ mutated complex (A85G) $3.1 \AA$, for G62U $3.2 \AA$, for A48G and G84A $3.1 \AA$ and for G33A $3.4 \AA$, respectively. The plots for RMSD versus time for wild type and all mutated complex is shown in Figure 1. For the wild type riboswitch, the RMSD values are similar up to $17 \mathrm{~ns}$ and decreased up to $21 \mathrm{~ns}$ and then subsequently increased. 

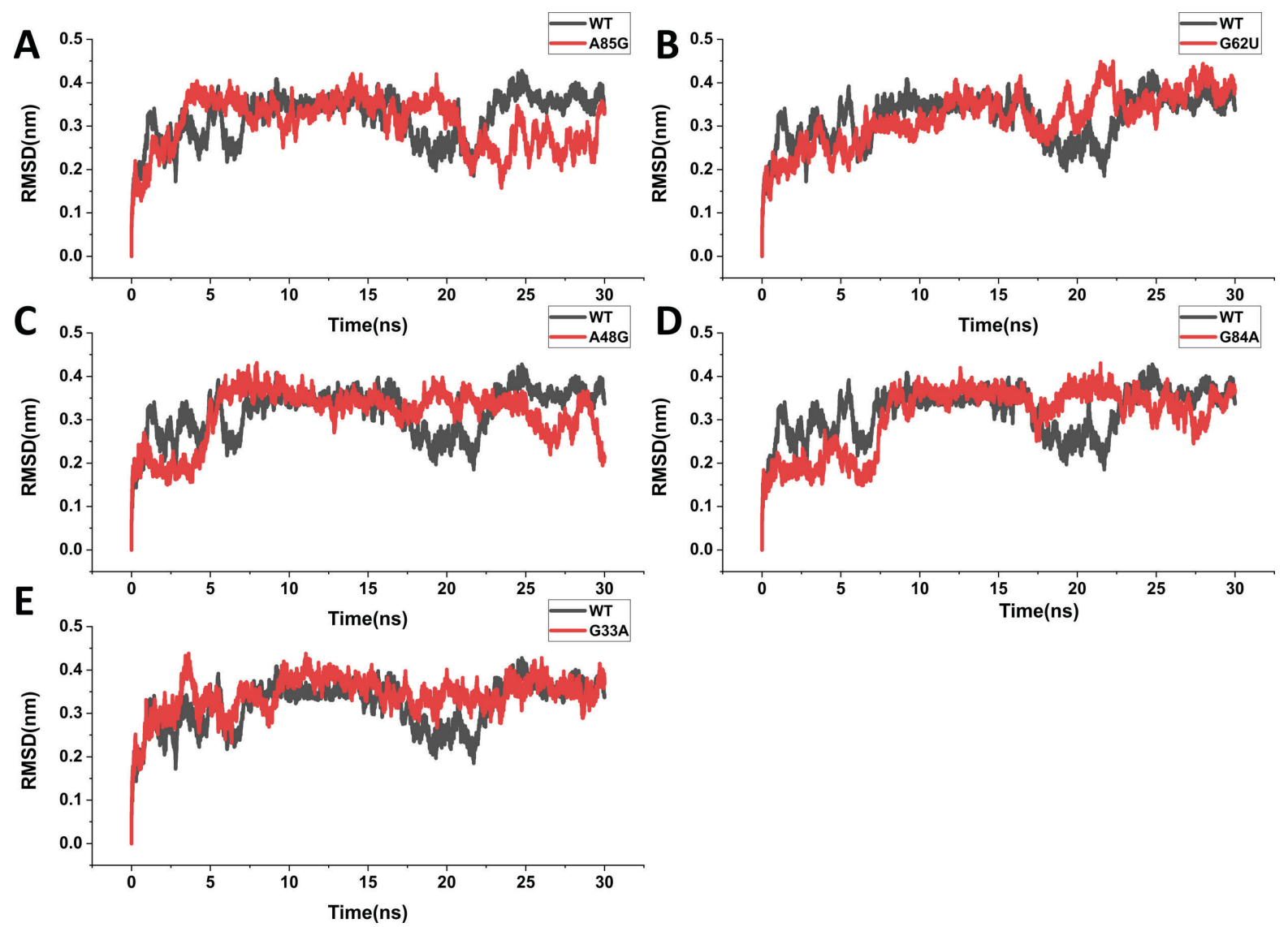

Figure 1. The root mean square deviation (RMSD) values are plotted versus simulation time for wild type and A85G, G62U, A48G, G84A, G33A mutants.

The root mean square fluctuations (RMSF) have been calculated for all complexes to study the flexible regions. For all complexes, wild type, as well as the mutants RMSF profile were found to be very similar (figure SI supporting information) and the regions at the two ends of the aptamer domain like residues 2-3, 51-61, 110-113 in the riboswitch structure are flexible in nature. To study the folding profile, the radius of gyration $(\mathrm{Rg})$ values have been calculated for 30ns MD simulation run (figure SIl supporting information). The radius of gyration was found to be similar for wild type and mutant complexes. This indicates that riboswitch structures do not undergo major conformational changes during simulation.

\section{Hydrogen Bonding Analysis}

A total number of hydrogen bonds between ligand and riboswitch have been calculated for all the complexes (Figure 2). It is clear from the profiles that the mutation A85G and G84A caused the decrease in the total number of the hydrogen bonds as compare to G62U, A48G, and G33A. The average number of hydrogen bond found to be 8.41 for wild type and for mutations A85G, G62U, A48G, G84A, and
G33A it is found to be $6.50,7.60,7.89,7.54$ and 8.11 respectively.

For further elucidation of interactions between ligand and binding site residues we prepared the trajectories

Table 1. Interactions involved in ligand binding with wild type as well as mutated riboswitch structure.

\begin{tabular}{ccccccc}
\hline Residue & Crystal str. & A85G & G62U & A48G & G84A & G33A \\
\hline G-10 & $2-\mathrm{HB}$ & $3-\mathrm{HB}$ & $2-\mathrm{HB}$ & $1-\mathrm{HB}$ & $3-\mathrm{HB}$ & $2-\mathrm{HB}$ \\
$\mathrm{G}-11$ & $3-\mathrm{HB}$ & $1-\mathrm{HB}$ & $2-\mathrm{HB}$ & $1-\mathrm{HB}$ & $2-\mathrm{HB}$ & $1-\mathrm{HB}$ \\
$\mathrm{G}-32$ & $1-\mathrm{HB}$ & - & $1-\mathrm{HB}$ & - & - & $1-\mathrm{HB}$ \\
$\mathrm{G}-33$ & $(\mathrm{a})$ & $(\mathrm{a})$ & $(\mathrm{a})$ & $(\mathrm{a})$ & $(\mathrm{a})$ & $(\mathrm{a})$ \\
$\mathrm{A}-48$ & $\pi-\pi$, & $\pi-\pi$, & $\pi-\pi$, & $\pi-\pi$, & $\pi-\pi$, & $\pi-\pi$, \\
$\mathrm{G}-62$ & $1-\mathrm{HB}$ & $1-\mathrm{HB}$ & $1-\mathrm{HB}$ & $1-\mathrm{HB}$ & $1-\mathrm{HB}$ & $1-\mathrm{HB}$ \\
$\mathrm{G}-84$ & $1-\mathrm{HB}$ & $1-\mathrm{HB}$ & - & $2 \mathrm{HB}$ & $1-\mathrm{HB}$ & $1-\mathrm{HB}$ \\
$\mathrm{A}-85$ & $\pi-\pi$ & $1-\mathrm{HB}$ & $1-\mathrm{HB}$ & - & $1-\mathrm{HB}$ & - \\
$\mathrm{A}-99$ & $1-\mathrm{HB}$ & - & $2-\mathrm{HB}$ & $2-\mathrm{HB}$ & $2-\mathrm{HB}$ & $2-\mathrm{HB}$ \\
\hline
\end{tabular}

(a) Mg mediated interaction. 


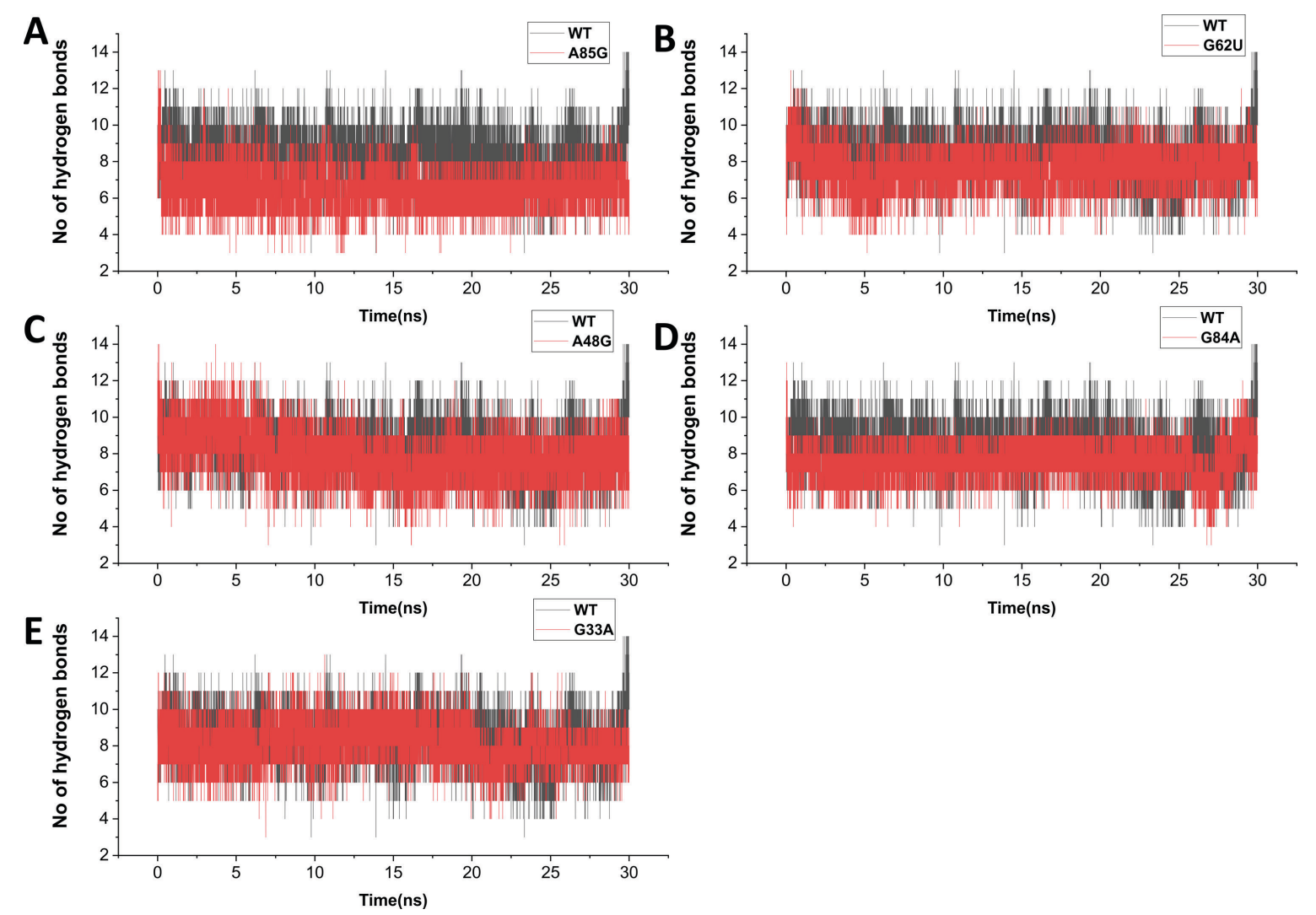

Figure 2. The total number of hydrogen bonds formed between FMN ligand with binding site residues for wild type and all mutated riboswitch complexes.

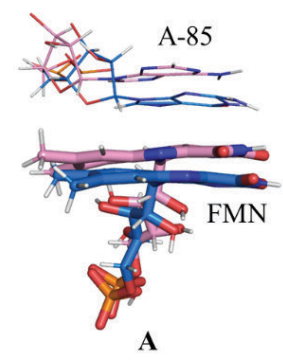

A

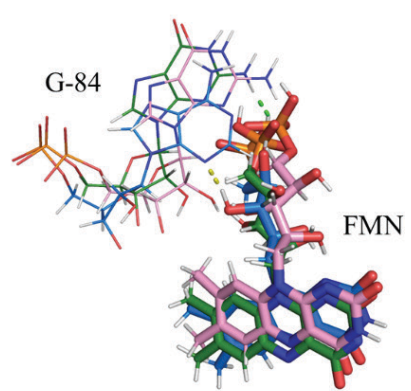

D

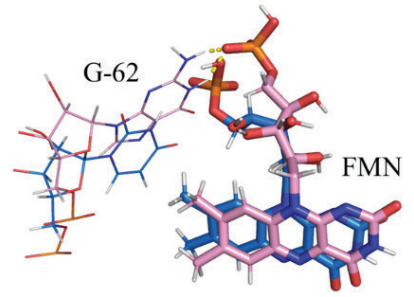

B

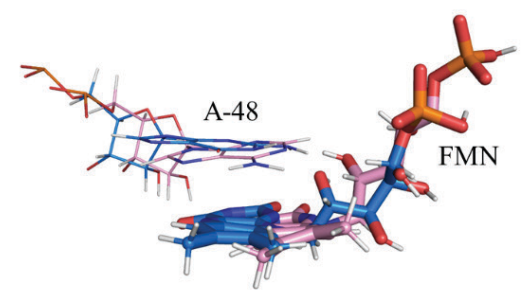

C

Figure 3. The wild type FMN riboswitch and mutated riboswitch are superimposed and shown in pink and blue color respectively. Ligand FMN is showed as sticks whereas residues are shown as lines. (A) A-85; (B) G-62; (C) A-48; (D) G-84; (E) G-33. 
from the 30 ns run and studied the binding site interactions for all complexes. (Table 1, Figure 3). From Table 1, it is clear that except the G-62 mutation all other mutated residues can maintain the hydrogen bonding with FMN ligand as the wild type.

In Figure 4A, the residue A-85 in wild type and mutated $\mathrm{G}-85$ showed as stacking with isoalloxazine ring in FMN ligand (Figure $3 \mathrm{~A}$ ). We have calculated the distance versus time between the $\mathrm{N} 10$ in $\mathrm{FMN}$ and $\mathrm{C} 5$ in $\mathrm{A} 85$ to study the interaction between $F M N$ and $A 85$ for wild type and mutant A85G (Figure $4 A$ and $5 A$ ). This indicates that these two rings come close to each other during the simulation and appears to be the result of $\pi-\pi$ interaction between FMN and A85. The electrophilic nature of N10 in FMN augments such stacking interactions. ${ }^{[36]}$ In the mutant G62U, the uracil loses the hydrogen bonding made by guanine with the phosphate group in FMN ligand (Figure 3B). In the wild type, the residue $A-48$ has $\pi-\pi$ stacking interaction with isoalloxazine ring in FMN ligand (Figure $3 \mathrm{C}$ ). In the mutant A48G, the distance between the C5A in FMN and N3 in A-48 is plotted with respect to time (Figure $4 B$ and $5 B$ ), and it is clear from the graph that this distance is shortened after such $A$ to $G$ mutation. G-84 forms a hydrogen bond with the oxygen of the phosphate group in the crystal structure

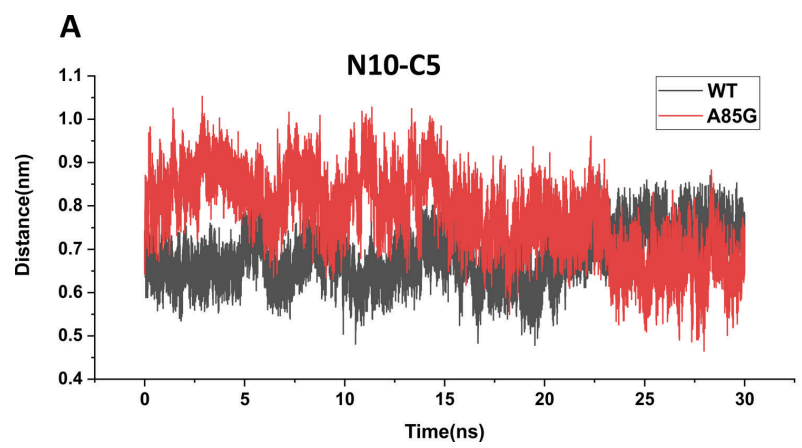

(green color residue Figure 3D) whereas, during the simulation, it does not exhibit this interaction (pink color residue and FMN in Figure 3D). However, when we mutated G-84 with $A-84$, it loses the interaction with the oxygen of the phosphate group but forms a new hydrogen bond with a hydroxyl group in the ribityl-phosphate chain in FMN. The residue $\mathrm{G}-33$ forms a magnesium mediated interaction with FMN ligand after mutation with A-33 it maintains the magnesium mediated interaction (Figure $3 \mathrm{E}$ ).

\section{Binding Energy Analysis}

The binding energy was calculated for wild type and all mutated ligand-riboswitch complexes with the Universal force field summarized in Table 2 . The calculated results indicate that the mutation of adenine at 48 position with guanine and mutation of G-62 with U-62 found to enhance

Table 2. Relative binding energy for wild type and all mutated complexes calculated with uff force field. (Values in $\mathrm{kcal} \mathrm{mol}^{-1}$ ).

\begin{tabular}{ccccccc}
\hline Type of energy & WT. & A85G & G62U & A48G & G84A & G33A \\
\hline Binding energy & 0.0 & 30.4 & -65.6 & -65.7 & -1.4 & -15.0 \\
\hline
\end{tabular}

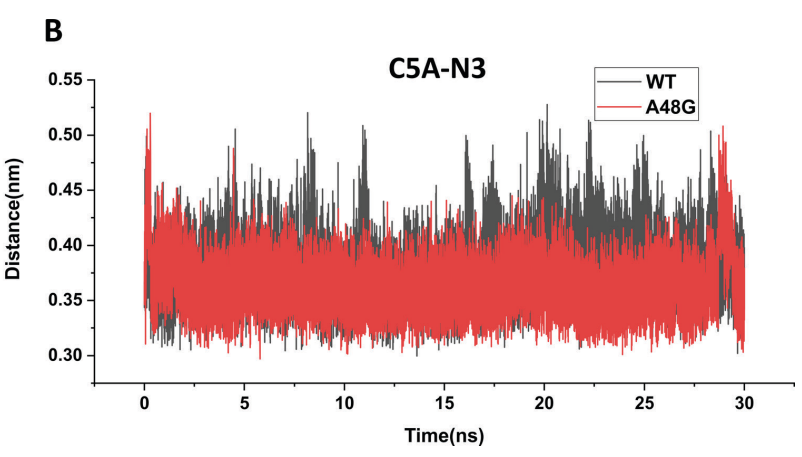

Figure 4. (A) Distance between the N10 in FMN and C5 in A-85 plotted versus time for wild type and mutant A85G; (B) Distance between the $\mathrm{C} 5 \mathrm{~A}$ in $\mathrm{FMN}$ and $\mathrm{N} 3$ in $\mathrm{A}-48$ with respect to time for wild type and mutant $\mathrm{A} 48 \mathrm{G}$.

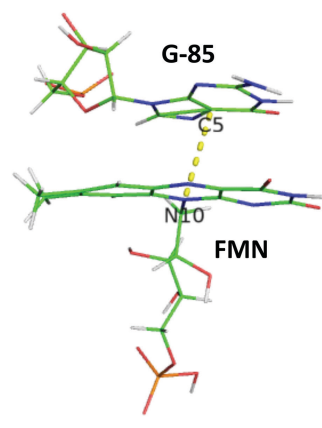

A

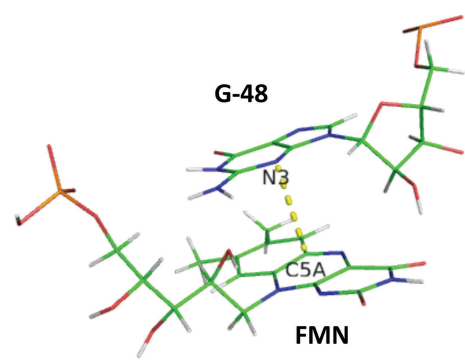

B

Figure 4. The atom numbers are indicated for A85G and A48G (A) N10 in FMN and C5 in G-85; (B) C5A in FMN and N3 in G-48. 
the binding energy. The mutation at G-84 and G-33 also found to enhance the binding energy as compared to the wild type. However, the mutation of A-85 found to be lower in binding energy than the wild type. These results suggest that the mutations performed contribute largely to stabilize the ligand binding with FMN riboswitch (Table 2).

\section{CONCLUSION}

In this work, we have examined the effect of a single mutation on the structural stability of FMN riboswitch with molecular dynamics (MD) simulations. We have chosen to mutate the binding site residues, A85G, G62U, A48G, G84A, and G33A in FMN riboswitch. The results obtained from MD simulations show similar profiles for RMSD, RMSF, and radius of gyration calculations for wild type and mutants. The average number of hydrogen bonds between the ligand and binding site residue are decreased for the mutated complexes in all cases. The results obtained from energy calculations suggest that the single point mutation in riboswitch structure affects the ligand binding energy. The relative binding energy for mutated FMN bound complexes at A48G and G62U was found to enhance the binding ability as compared to wild type FMN riboswitch. The mutant A85G showed the lowest number of average hydrogen bonds (6.50) as compared to wild type (8.41) and other mutants, and the binding energy was also found to weaker in this case. Overall, these results suggest that the mutation of adenine with guanine is favored to improve the binding affinity of the FMN with FMN riboswitch and this is due to more number of polar functional groups in the later residue. These results shed light on the role of mutants to enhance the binding affinity of ligands with FMN riboswitch and that can serve in the preparation of new ligands with better efficacy for antibacterial applications.

Acknowledgment. P. D. W. acknowledges CSIR, New Delhi, India, for the GATE-junior research fellowship and P. D. W. thankful to AcSIR for her Ph.D. registration. The present computational work is supported by Department of Science and Technology, Government of India, under grant no. EMR/2017/004652. B.G. thanks DBT (Grant no. BT/PR12730/BID/7/523/ 2015) New Delhi for financial support. We thank the reviewers for their valuable comments and suggestions that have helped us to improve the paper.

Supplementary Information. Supporting information to the paper is attached to the electronic version of the article at: http://doi.org/10.5562/cca3550.

PDF files with attached documents are best viewed with Adobe Acrobat Reader which is free and can be downloaded from Adobe's web site.

\section{REFERENCES}

[1] J. R. Mellin, P. Cossart, Trends Genet. 2015, 31, 150156. https://doi.org/10.1016/j.tig.2015.01.005

[2] Q. V. Vicens, J. Berman, H. Kaur, K. W. Kells, P. Wickens, R. C. Gadwood, H. J. Schostarez, R. K. Suto, K. F: Blount, R. T. Batey, ACS Chem Biol 2018, 13, 2908-2919.

https://doi.org/10.1021/acschembio.8b00533

[3] S. V. Harbaugh, M. E. Davidson, Y. G. Chushak, N. Kelley-Loughnane, M. O. Stone, Proc. SPIE 2008, 7040, 70400C.

[4] D. Matzner, G. J. Mayer, Med. Chem 2015, 58, 32753286. https://doi.org/10.1021/jm500868e

[5] E. R. Lee, K. F. Blount, R. R. B. RNA Bio/ 2009, 6, 187194. https://doi.org/10.4161/rna.6.2.7727

[6] K. F. Blount, C. Megyola, M. Plummer, D. Osterman, T. O'Connell, P. Aristoff, C. Quinn, R. A. Chrusciel, T. J. Poel, H. J. Schostarez, C. A. Stewart, D. P. Walker, P. G. Wuts, R. R. Breaker, Antimicrob. Agents Chemother 2015, 59, 5736-5746.

https://doi.org/10.1128/AAC.01282-15

[7] J. A. Howe, H. Wang, T. O. Fischmann, C. J. Balibar, L. Xiao, A. M. Galgoci, J. C. Malinverni, C. Juliana, T. Mayhood, A. Villafania, A. Nahvi, N. Murgolo, C. M. Barbieri, P. A. Mann, D. Carr, E. Xia, P. Zuck, D. Riley, R. E. Painter, S. S. Walker, B. Sherborne, R. de. Jesus, W. Pan, M. A. Plotkin, J. Wu, D. Rindgen, J. Cummings, C. G. Garlisi, R. Zhang, P. R. Sheth, C. J. Gill, H. Tang, T. Roemer. Nature 2015, 526, 672-677. https://doi.org/10.1038/nature15542

[8] A. Serganov, L. Huang, D. J. Patel, Nature 2009, 458, 233-237. https://doi.org/10.1038/nature07642

[9] A. B. Rode, T. Endoh, N. Sugimoto, Angew. Chemie Int. Ed. 2015, 54, 905-909.

https://doi.org/10.1002/anie.201407385

[10] A. B. Rode, T. Endoh, N. Sugimoto, Bull. Chem. Soc. Jpn. 2015,88, 946-948.

https://doi.org/10.1246/bcsj.20150063

[11] Y. Li, M. D. Netherland, C. Zhang, H. Hong, P. Gong, PLoS One 2019,14, e0216116.

https://doi.org/10.1371/journal.pone.0216116

[12] P. Kar, V. Knecht, J. Phys. Chem B 2012, 116, 61376149. https://doi.org/10.1021/jp3022612

[13] W. Jingyuan, C. Liangliang, C. Yinchu, L. Yan, J. Honghua, Z. Hua, W. Ping, Bioengineered 2019,10, 142-149. https://doi.org/10.1080/21655979.2019.1607710

[14] L. Zhao, T. Sun, J. Pei, Q. Ouyang, Proc. Natl. Acad. Sci 2015,112, E4046-E4054. https://doi.org/10.1073/pnas.1502126112 
[15] V. Assadollahi, B. Rashidieh, M. Alasvand, A. Abdolahi, J. A. Lopez, J. Cell. Biochem 2019, 120, 13046. https://doi.org/10.1002/jcb.28575

[16] P. D. Wakchaure, J. Kalyanashis, G. Bishwajit, J. Biomol. Struct.Dyn. 2019, 37, 1-14. https://doi.org/10.1080/07391102.2019.1665587

[17] H. M. Berman, T. Battistuz, T. N. Bhat, W. F. Bluhm, P. E. Bourne, K. Burkhardt, Z. Feng, G. L. Gilliland, L. lype, S. Jain, P. Fagan, J. Marvin, D. Padilla, V. Ravichandran, B. Schneide, N. Thanki, H. Weissig, J. D. Westbrook, C. Zardecki, Acta Crystallogr. Sect. D Biol. Crystallogr 2002, 58, 899-907. https://doi.org/10.1107/S0907444902003451

[18] Q. Vicens, E. Mondragón, R. T. Batey, Nucleic Acids Res 2011,39, 8586-8598.

https://doi.org/10.1093/nar/gkr565

[19] L. L. C. Schrödinger, The PyMOL Molecular Graphics System, Version 1.8. 2015.

[20] D. S. San Diego, BIOvIA, Discovery studio modeling environment.

[21] A. W. Sousa, W. F. Vranken, ACPYPE - AnteChamber PYthon Parser interfacE. BioMed Cent. Ltd 2012, 5, 1. https://doi.org/10.1186/1756-0500-5-367

[22] A. Jakalian, D. B. Jack, C. I. Bayly, J. Comput. Chem 2002, 23, 1623-1641. https://doi.org/10.1002/jcc.10128

[23] M. J. Abraham, T. Murtola, R. Schulz, S. Páll, J. C. Smith, B. Hess, E. Lindah, SoftwareX 2015, 1, 19-25. https://doi.org/10.1016/j.softx.2015.06.001

[24] H. J. C. Berendsen, J. P. M. Postma, W. F. Van Gunsteren, A. Dinola, J. R. Haak, J. Chem. Phys 1984, 81, 3684. https://doi.org/10.1063/1.448118

[25] H. J. C. Berendsen, D. van der Spoel, R. van Drunen, Comput. Phys. Commun 1995, 91, 43-56. https://doi.org/10.1016/0010-4655(95)00042-E
[26] A. T. Guy, T. J. Piggot, S. Khalid, Biophys J. 2012, 103, 1028-1036. https://doi.org/10.1016/j.bpj.2012.08.012

[27] S. P. Pronk, R. Schulz, P. Larsson, P. Bjelkmar, R. Apostolov, M. R. Shirts, J. C. Smith, P. M. Kasson,D. van.der Spoel, B. Hess, E. Lindahl, Bioinformatics 2013, 29, 845-854.

https://doi.org/10.1093/bioinformatics/btt055

[28] B. Hess, H. Bekker, H. J. C. Berendsen, J. G. E. M. Fraaije, J. Comput. Chem 1997, 18, 1463-1472. https://doi.org/10.1002/(SICI)1096987X(199709)18:12<1463::AID-JCC4>3.3.CO;2-L

[29] T. Darden, D. York, L. Pedersen, J. Chem. Phys 1993, 98, 10089. https://doi.org/10.1063/1.464397

[30] Origin (OriginLab, Northampton, MA).

[31] A. K. Rappé, W. A. Goddard, J. Phys. Chem 1991,95, 3358-3363. https://doi.org/10.1021/j100161a070

[32] G. E. S. M. J. Frisch, G. W. Trucks, H. B. Schlegel, B. M. M. A. Robb, J. R. Cheeseman, G. Scalmani, V. Barone, H. P. H. G. A. Petersson, H. Nakatsuji, M. Caricato, X. Li, M. H. A. F. Izmaylov, J. Bloino, G. Zheng, J. L. Sonnenberg, T. N. M. Ehara, K. T. Gaussian, Inc., Wallingford CT. 2013.

[33] F. Lipparini, G. Scalmani, B. Mennucci, Phys.Chem. Chem.Phys. 2009, 48, 11617-11623. https://doi.org/10.1039/b915898g

[34] K. Dedachi, Y. Ishikawa, T. Nakatsu, T. Natsume, J. Mol. Struct. THEOCHEM 2008, 854, 70-75. https://doi.org/10.1016/j.theochem.2007.12.039

[35] T. Sasami, R. Tawarada, A. Ohkubo, M. Sekine,K. Seio, J. Mol. Struct. THEOCHEM 2009, 899, 54-60. https://doi.org/10.1016/j.theochem.2008.12.012

[36] T. Tandarić, R. Vianello, ACS Chem. Neurosci. 2019, 10, 3532-3542. https://doi.org/10.1021/acschemneuro.9b00147 